\title{
AUTOMATIC LIVE AND DEAD CELL CLASSIFICATION VIA HYPERSPECTRAL IMAGING
}

\author{
He Chen ${ }^{1}$ Benjamin Ho ${ }^{2}$ Haofei Wang ${ }^{3} \quad$ SayHwa Tan ${ }^{2}$ Chun-Xia Zhao ${ }^{3}$ Nam-Trung Nguyen ${ }^{1}$ \\ Yongsheng Gao ${ }^{4}$ Jun Zhou ${ }^{\text {* }}$ \\ ${ }^{1}$ School of Information and Communication Technology, Griffith University, Nathan, Australia \\ ${ }^{2}$ Queensland Micro and Nanotechnology Centre, Griffith University, Nathan, Australia \\ ${ }^{3}$ Australian Institute for Bioengineering and Nanotechnology, The University of Queensland, St. Lucia, Australia \\ ${ }^{4}$ School of Engineering, Griffith University, Nathan, Australia
}

\begin{abstract}
Classification of live and dead human ovarian cancer cells (SKOV3) is an important task in biomedicine research. Fluorescence is the most common technique to distinguish live and dead cells. However, it requires physical contact to the cells, which affects the appearance of the cells and their features to a certain degree. There is an urgent need to develop a novel non-invasive technology for this application. This paper proposes the first hyperspectral image (HSI) based system to address this issue. A microscopic hyperspectral imaging system is built to capture cell images. Then morphological methods are employed to extract spectralspatial features for an SVM classifier that is capable of automatically distinguishing live and dead SKOV3 cells. We show that tensor morphology profile (TMP) leads to the best discriminative capability when compared to other features. At the same time, our work also shows that HSI works better than RGB images for this novel application thanks to its fine spectral information.
\end{abstract}

Index Terms - hyperspectral imaging, cell classification, mathematical morphology, tensor modelling

\section{INTRODUCTION}

Hyperspectral image classification is drawing increasing interests in many fields, including biomedicine [1], food [2], agriculture [3] and remote sensing [4]. New methods and applications are explored to take the most advantages of the rich information that HSIs provide both spatially and spectrally.

In the field of biomedicine, researchers have been utilizing HSI technologies to improve the performance of cell classification. For example, Chen et al. [5] proposed a hyperspectral images classification framework that integrates Gabor filtering with convolutional neural network (CNN).
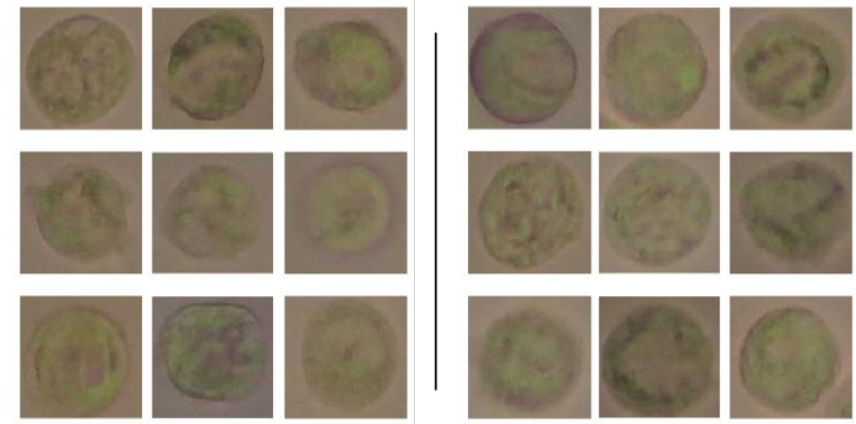

Figure 1: Color images of dead cells (left) and live cells (right).

Qiong et al. [6] explored spatial context for an extreme learning machine with Markov random field.

However, to the best of our knowledge, there is no research on classifying live and dead cells with the assistance of HSI. Currently, the most common method for this task is labelling cells using fluorescent dyes and then put them under light of certain wavelength to have the fluorescent reaction before distinguishing cells. Such method may damage the cells and result in substantial change to the appearance or features of cells compared with those in the natural environment. Furthermore, the process itself is costly and non-consistent due to manual operations.

Cell death is normally associated with morphological changes such as cell membrane blebbing, dilated organelles, and condensed nucleus [7] [8]. In addition, dead cells show lower forward scatter and higher side scatter compared to live cells [9]. However, these fine changes are hardly recognizable to the naked eyes even with the help of microscopy or flow cytometer instrument (See Figure 1 for examples). Nevertheless, this biological analysis points out a path to explore morphological hyperspectral image processing to distinguish the fine changes between live and dead cells by exploring both spatial and spectral morphological features.

\footnotetext{
* Corresponding author.
} 
Mathematical morphology (MM) is the foundation of morphological image processing. Since it was introduced for the HSI classification [10], a number of extensions have been developed to better characterize the spectral spatial properties of targets. Typical works include extended morphology profile (EMP) [11] and its tensor-based version tensor morphology profile (TMP) [12]. In particular, TMP addresses the ordering problem in EMP and made it more applicable to high-dimensional data processing.

In this paper, we introduce the first system that uses hyperspectral imaging to distinguish live and dead cells. Similar to the traditional remote sensing image classification pipelines [13], our method follows the procedures of image preprocessing, feature extracting and classification. Among these steps, the quality of features plays an important role that influences the final classification accuracy. Therefore, we employ TMP which is one of the best spectral-spatial morphological features available. We compare it against several spectral-spatial features to showcase the effectiveness of this feature and our system. We also demonstrate the advantage of hyperspectral images over RGB images on this novel application.

\section{CELL IMAGING SYSTEM}

This proposed cell imaging system consists of both hardware and software components to capture the raw HSI images. Hardware components include a microscope, a hyperspectral camera, a camera controller and a high-performance desktop, as shown in Figure 2. The software components include a Windows 10 operation system and a program to control the camera.

\subsection{Hardware Components}

The core part of this hardware system is the hyperspectral camera, which is an IMEC SNAPSCAN VNIR B150U hyperspectral camera. It integrates a linescan HSI sensor based on CMOSIS CMV2000 image sensor. It has up to $3650 * 2048$ spatial resolution and $470-900 \mathrm{~nm}$ spectral range with 150 spectral bands. Furthermore, this camera sensor is integrated with cut-off filters directly to simplify the usage of camera. In the system, the camera is mounted onto the microscope. Along with the camera, a controller is used to control the complicated settings of the camera.

Another main component in this system is the Nikon Eclipse TS100 microscope which is a compact highperformance microscope. It provides environment friendly LED light source to our cameras. With an easy-to-rotate nosepiece, several objectives can be easily mounted and switched. In our system, we use a 20x objective lens.

Furthermore, a high-performance desktop is also necessary in the system. Since an image captured by this camera can be as large as $3 \mathrm{GBs}$, it requires a powerful $\mathrm{CPU}$

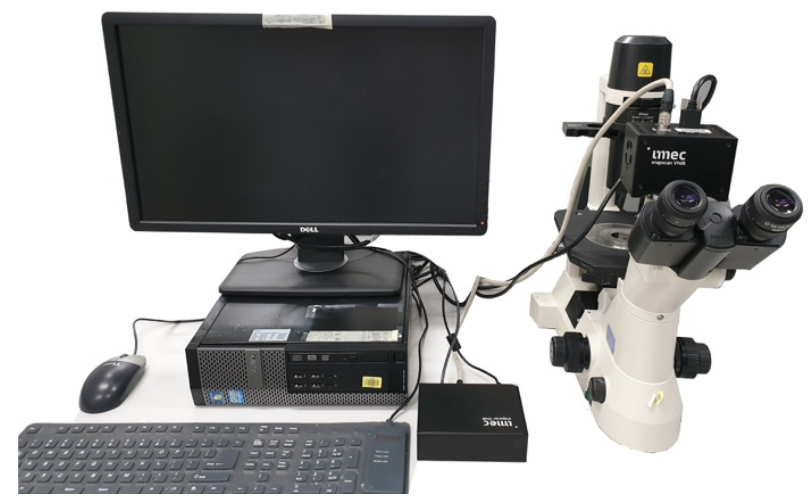

Figure 2: Cell Imaging System.

and a USB3.0 port and cable to connect the camera. In our system, we use a customised desktop, which has Intel $®$ Core $^{\mathrm{TM}}$ i7-7800X CPU@3.50GHz and 128 GB memory.

\subsection{Software Components}

The hyperspectral camera in our system is controlled by its SNAPSCAN program to acquire hyperspectral data cubes, generating a real-time view and exporting the data. It has a few parameters to adjust, including binning columns, binning rows, integration time, analog time, scanning mode, scanning direction, scanning velocity and so on. It can export the data into four formats, which are ENVI, TIFF, JPG and BMP respectively. With all the flexible options, this software can adjust the hardware to the best possible condition under every circumstance.

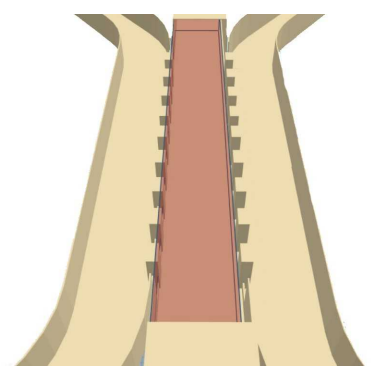

Figure 3: The microfluidic device is composed of three parallel microchannels separated by arrays of rectangular pillars and in the size of micrometres. The presence of micropillars facilitates confinement of gel solution within the middle channel in brown, while independent fluid flow is allowed in both side channels in yellow.

\section{CELL DATASET}

We use human ovarian cancer cells for the experiments because this type of cells is widely used in tumour related research, so the viability assay is frequently conducted in lab. 
The live samples are collected and suspended in phosphate buffered saline (PBS). Dead samples are prepared by treating live cells with $2 \%$ acetic acid for 5 minutes at room temperature, followed by 3 times wash using PBS to remove the acid solution. When the cells are ready, we use the aforementioned HSI camera and a Canon G1X to take the HSI and color images, respectively.

Due to the hardly visible differences between the dead and live cells to naked eyes, to enable generation of the cell ground-truth labels, we conduct the experiment in the microfluidic device as shown in Figure 3. It has three channels, which hold live samples, collagen gel and dead samples respectively. Prior to cell loading into the microfluidic device, cold collagen gel solution is injected in the middle channel and allowed to polymerize for 30 minutes at $37^{\circ} \mathrm{C}$. After confirming that the gel solution is solidified, live and dead cell samples are injected into the left and right channels respectively. The collagen gel works as semipermeable membrane here. It allows PBS to flow freely from two sides, but it blocks the exchange between live and dead cells. Under such setting, we make sure that the samples are sitting in the same environment. This setting also allows the ground truth label for each cell to be conveniently generated. In total, we collect 405 live cells and 291 dead cells images, including both hyperspectral and color images. The mean spectral responses of live and dead cell samples are shown in Figure 4.

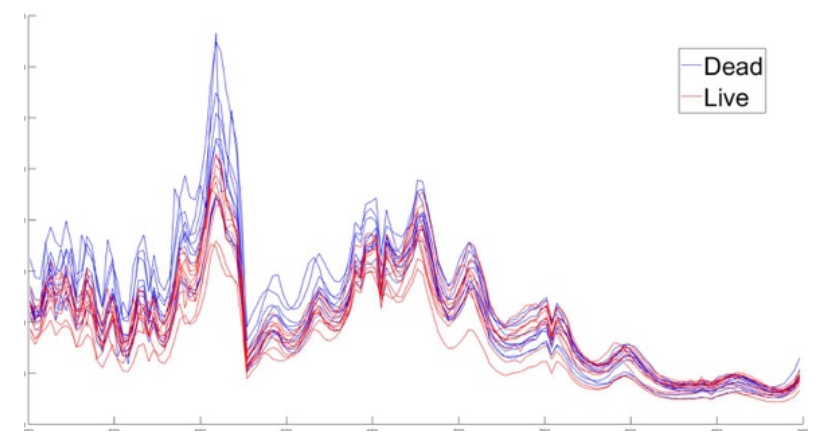

Figure 4: Illustration of mean spectral responses of live and dead cell samples.

\section{CELL CLASSIFICATION METHOD}

In this section, we introduce our image preprocessing and feature extraction method. We first preprocess the raw images with several calibration steps. Then we introduce TMP as the feature extraction method which is commonly used to process HSI data and get the 3D feature cubes. At last, the features are fed into a support vector machine (SVM) for training and testing purposes.

\subsection{Image preprocessing}

To extract each cell from the raw images, pseudo color images are first created based on the HSI data cubes to visualize the raw data. Then the cells are manually segmented into individual ones using Image Labeler APP in Matlab. Each cell is associated with a live/dead label. With all the extracted cell data cubes, a series of calibrations, including dark calibration and image size normalization, are conducted to make them standardized for the following steps of feature extraction.

\subsection{Feature Extraction}

In this system, a key step is to explore the morphological features from hyperspectral images for cells. To this end, we employ tensor morphology profile.

When dealing with hyperspectral images, tensor method is a powerful tool to represent the 3D cubes [15]. Its notation is commonly defined as:

$$
\mathcal{T} \in \mathbb{R}^{n_{1} * n_{2} * n_{3}}
$$

where $n_{1}$ and $n_{2}$ are the height and width of HSI images, $n_{3}$ is the number of the bands. Then HSI images can be described using tensor notation as:

$$
\mathcal{T}: S_{\mathcal{T}} \subset R^{n_{1} \times n_{2} \times n_{3}} \rightarrow\left\{0,1, \ldots, i_{\text {max }}\right\}
$$

where $S_{\mathcal{T}}$ is the object space for tensor.

Compared with EMP, the essential contribution of TMP is treating data cube as an $n$-dimension data rather than $n$ separate two-dimension images. We aim at extracting the cross-section cube of the n-dimension data $C_{h} I(x)$ :

$$
C_{h} I(x)= \begin{cases}0 & \text { if } I(x)<h \\ 1 & \text { if } I(x) \geq h\end{cases}
$$

where $I(x)$ is a two-dimension band image of the hyperspectral cube and $h$ is a threshold. When TMP processes the data, the threshold goes through the intensity set $\left\{0,1, \ldots, i_{\max }\right\}$ to get multiple results of $\mathrm{n}$-dimension data. Then the results can be used to perform morphological operation to the original n-dimension data as the implementation of filter elements in binary morphology [12]. Thus, dilation, erosion, opening and closing operations can be formulated respectively as:

$$
\begin{aligned}
& (\mathcal{T} \oplus b)(x)=\sup _{y \in E}[\mathcal{T}(y)+b(x-y)] \\
& (\mathcal{T} \ominus b)(x)=\inf _{y \in E}[\mathcal{T}(y)-b(y-x)] \\
& \mathcal{T} \circ b=(\mathcal{T} \ominus b) \bigoplus b \\
& \mathcal{T} \cdot b=(\mathcal{T} \oplus b) \ominus b
\end{aligned}
$$


where $b$ is the element of filter, which is the set of results from the operation in equation (3) with different thresholds.

With all the definition above, we can finally implement the step to get the spatial-spectral feature from an ndimension data using:

$$
\widehat{H}_{p}(\mathcal{T})=\left[H_{1}(\mathcal{T}), H_{2}(\mathcal{T}), \ldots, H_{p}(\mathcal{T})\right]
$$

where $H_{p}(\mathcal{T})$ is a series of morphological operations on the tensor $\mathcal{T}$.

To classify the HSI cubes of live and dead cells, each set of final results of $\widehat{H}_{p}(\mathcal{T})$ will be fed into a liner SVM [16] to make the prediction.

\section{EXPERIMENTS AND RESULTS}

We conducted two experiments to compare several spectralspatial features and classification performance under HSI and color images. The features under comparison included SIFT3D [17], 3DLDP [18] and SS_SIFT [19], EMP [11] and TMP [12]. Among them, SIFT3D was developed to extract a robust descriptor based on the classic scale-invariant feature transform (SIFT) method. It was originally used for abdominal CT images. 3DLDP was used for human face recognition based on local derivative pattern descriptor. SS_SIFT is another method developed from the SIFT. It explores both spatial and spectral dimensions simultaneously to extract the features. Since SIFT3D, 3DLDP and SS_SIFT were developed for higher dimensional data only, we did not include their results on the RGB images.

We implemented the cell classification methods using Matlab, including the code for image preprocessing, feature extraction, and classification. LIBSVM was used as the SVM classifier [16].

Table 1: Accuracy of the classification results using different features on HSI and RGB data.

\begin{tabular}{|l|c|c|c|c|c|}
\hline & $\begin{array}{c}\text { SIFT3D } \\
{[17]}\end{array}$ & $\begin{array}{c}\text { 3DLDP } \\
{[18]}\end{array}$ & $\begin{array}{c}\text { SS_SIFT } \\
{[19]}\end{array}$ & $\begin{array}{c}\text { EMP } \\
{[11]}\end{array}$ & $\begin{array}{c}\text { TMP } \\
{[12]}\end{array}$ \\
\hline HSI & $72.68 \%$ & $81.53 \%$ & $77.81 \%$ & $74.96 \%$ & $\mathbf{8 3 . 7 2 \%}$ \\
\hline RGB & - & - & - & $57.16 \%$ & $79.85 \%$ \\
\hline
\end{tabular}

\subsection{Results and Analysis}

Table 1 reports the mean accuracy after randomly splitting the collected data for 10 times in training and testing. Two observations can be made from Table 1. First, HSI based classification system has clearly outperformed color image based system. TMP achieved $3.87 \%$ higher accuracy on HSI dataset than on color images, while EMP achieved more significant accuracy improvement using HSI. The reason is hyperspectral images contain more detailed spectral-spatial information than the color images, which not only allows effective analysis of the spatial structure pattern, but also provides the opportunity to analyse the spectral features.

When features are concerned, TMP has produced the best performance on the HSI dataset among all the feature extraction methods. It is clear that TMP takes more advantages of the characteristics of HSI images than the others due to its powerful morphological feature description capability. Since EMP only processes the cross-section image at each wavelength separately and then stacks them together, it only extracted a number of spatial features and ignored their internal spectral correlation. However, with the help of tensor, TMP treats the data cube as one whole piece of highdimension data and keeps the internal correlations between different spectral bands, making it more powerful in characterizing the joint spectral-spatial information.

\section{CONCLUSION}

This paper described a novel approach for live and dead cells assay using hyperspectral imaging technology. This is the first work that conducts automatic live and dead cells assay using spectral-spatial information with morphological analysis on hyperspectral images. The experimental results show that HSI is very promising in discriminating live/dead cells which is very difficult for human to make visual interpretation. Among all compared spectral-spatial features, tensor morphology profile has achieved the best performance due to its superior high dimensional feature extraction capability. The advantage of the proposed system also comes from enabling cells assay without any physical contact to cells of fluorescent dyes. This may generate potentially profound influence on the cell assay task by increasing the efficiency and reducing the cost effectively.

\section{REFERENCES}

[1] G. Lu, B. Fei, "Medical hyperspectral imaging: a review," Journal of biomedical optics, Vol. 19, No. 1, 010901, 2014.

[2] S. Marshall, T. Kelman, T. Qiao, P. Murray and J. Zabalza, "Hyperspectral imaging for food applications," 23rd European Signal Processing Conference (EUSIPCO), pp. 2854-2858, 2015.

[3] A. Lowe, N. Harrison and A. P. French, "Hyperspectral image analysis techniques for the detection and classification of the early onset of plant disease and stress," Plant Methods, 13:80, 2017.

[4] F. I. Alam, J. Zhou, A. W. Liew, X. Jia, J. Chanussot and Y. Gao. "Conditional random field and deep feature learning for hyperspectral image classification," IEEE Transactions on Geoscience and Remote Sensing, Vol. 57, No. 3, pp. 1612-1628, 2019. 
[5] Y. Chen, L. Zhu, P. Ghamisi, X. Jia, G. Li and L. Tang, "Hyperspectral images classification with Gabor filtering and convolutional neural network," IEEE Geoscience and Remote Sensing Letters, Vol. 14, No. 12, pp. 2355-2359, 2017.

[6] Q. Ran, L. Chang, W. Li, and X. Xu, "Spatial-spectral blood cell classification with microscopic hyperspectral imagery," Optical Spectroscopy and Imaging, Vol. 10461, 0146102, 2017.

[7] S. A. Rahman, N. A. Wahab and S. N. B. Malek, "In vitro morphological assessment of apoptosis induced by antiproliferative constituents from the rhizomes of curcuma zedoaria," Evidencebased Complementary and Alternative Medicine, 257108, 2013.

[8] S. Rello, J. C. Stockert, V. Moreno, A. Gamez, M. Pacheco, A. Juarranz, M. Canete and A. Villanueva, "Morphological criteria to distinguish cell death induced by apoptotic and necrotic treatments," Apoptosis, Vol. 10, No. 1, pp. 201-208, 2005.

[9] Z. Darzynkiewicz, E. Bedner, "Analysis of apoptotic cells by flow and laser scanning cytometry," Methods in Enzymology, Vol. 322, pp. 18-39, 2000.

[10] A. Plaza, P. Martinez, R. Perez and J. Plaza, "Spatial/spectral endmember extraction by multidimensional morphological operations," IEEE Transactions on Geoscience and Remote Sensing, Vol. 40, No. 9, pp. 2025-2041, 2002.

[11] J. A. Benediktsson, J. A. Palmason, and J. R. Sveinsson, "Classification of hyperspectral data from urban areas based on extended morphological profiles," IEEE Transactions on Geoscience and Remote Sensing, Vol. 43, No. 3, pp. 480-491, 2005.

[12] J. Liang, J. Zhou, and Y. Gao, "Tensor morphological profile for hyperspectral image classification," IEEE International Conference on Image Processing, pp. 2197-2201, 2016

[13] C. Liu, J. Zhou, J. Liang, Y. Qian, H. Li and Y. Gao, "Exploring structural consistency in graph regularized joint spectral-spatial sparse coding for hyperspectral image classification," IEEE Journal of Selected Topics in Applied Earth Observations and Remote Sensing, Vol. 10, No. 3, pp. 1151-1164, 2017.

[14] F. Liu, J. V. Velikina, W. F. Block, R. Kijowski and A. A. Samsonov, "Fast realistic MRI simulations based on generalized multi-pool exchange tissue model," IEEE Transactions on Medical Imaging, Vol. 36, No. 2, pp. 527-537, 2017.

[15] S. Velasco-Forero and J. Angulo, "Classification of hyperspectral images by tensor modeling and additive morphological decomposition," Pattern Recognition, Vol. 46, No. 2, pp. 566-577, 2013.

[16] C. Chang and C. Lin, "LIBSVM: a library for support vector machines," ACM Transactions on Intelligent Systems and Technology, Vol. 2, No. 3, Article 27, 2011.
[17] B. Rister, M. A. Horowitz and D. L. Rubin, "Volumetric image registration from invariant keypoints," IEEE Transactions on Image Processing, Vol. 26, No. 10, pp. 4900-4910, 2017.

[18] J. Liang, J. Zhou and Y. Gao, "3D local derivative pattern for hyperspectral face recognition," IEEE International Conference and Workshops on Automatic Face and Gesture Recognition, pp. 1-6, 2015.

[19] S. L. Al-khafaji, J. Zhou, A. Zia and A. W. Liew, "Spectralspatial scale invariant feature transform for hyperspectral images," IEEE Transactions on Image Processing, Vol. 27, No. 2, pp. 837 $850,2018$. 